\title{
Photonic Tuning of Nanophosphor Transparent thin films
}

\author{
Gabriel Lozano, Dongling Geng, Hernán Míguez \\ Instituto de Ciencia de Materiales de Sevilla, Consejo Superior de Investigaciones \\ Cientificas-Universidad de Sevilla (CSIC-US), Américo Vespucio 49, 41092 Sevilla, Spain \\ Authore-mail address: g.lozano@ csic.es
}

\begin{abstract}
Chromaticity coordinates of $\mathrm{Dy}^{3+}$ - based nanocrystals deposited as thin films are modulated from blue to yellow using photonic multilayers that are transparent in the UV and resonant in the visible part of the electromagnetic spectrum.
\end{abstract}

OCIS codes:

\section{Introduction}

Phosphors with particle sizes smaller than $100 \mathrm{~nm}$ and controlled shapes opened the door to large-scale and low-cost fabrication of versatile transparent emitting thin films based on rare earth-doped nanocrystals. In particular, techniques such as screen printing, dip coating, and electrophoretic methods combined with high-temperature annealing or cold isostatic pressing have been reported to achieve transparent nanophosphor films with thicknesses on the order of the micrometer, which enable the development of photonic-based strategies to mold the photo luminescence process that seek to be herein investigated.

\section{Discussion}

We demonstrate that optical resonators allow tailoring the color hue of nanophosphor thin films, opening a route toward the development of new types of photoluminescence layers. Among those rare earth ions that are attractive for the realization of white light emission in a single phase host, $\mathrm{Dy}^{3+}$-doped materials are interesting because they show two emission bands in the blue and yellow spectral regions, being the intensity of the latter tunable through a careful selection of the inorganic host in which $\mathrm{Dy}^{3+}$ ions are incorporated. In particular we make use of $\mathrm{GdVO}_{4}$ as an inorganic host since this matrix provides the phosphor with chemical stability and large absorption cross section in the UV. A solvothermal method is employed to synthesize $\mathrm{GdVO}_{4}: \mathrm{Dy}^{3+}$ nanoparticles of controlled size and shape. We find the experimental conditions to process these nanophosphors in the shape of films of controlled thickness with excellent optical properties. To do this, different material properties including surface morphology, optical transparency, along with the static and dynamic photoemission spectra of RE films annealed at different temperatures are evaluated. As a result, we find the sintering conditions that yield nanophosphor coatings emitting most efficiently while preserving transparency. Finally, a series of photonic multilayers made of $\mathrm{ZrO}_{2}$ and $\mathrm{SiO}_{2}$ that integrate such nanophosphor films as optical cavities are designed and fabricated using solution processing techniques. Tuning the lattice parameter of the photonic multilayer allows tailoring the color of the photoemission of the $\mathrm{Dy}^{+}{ }^{+}$nanophosphor from green to blue or yellow with unprecedented precision.[1]

\section{Conclusions}

The emission color of $\mathrm{GdVO}_{4}: \mathrm{Dy}^{3+}$ nanocrystals can be strongly modulated from blue to yellow, using photonic multilayers, in which a transparent and bright nanophosphor film is integrated as an optical dopant. Photonics offers new opportunities to tailor the emission of nanophosphor materials, which can provide benefits to next generation light-emitting devices.

\section{References}

